\title{
An Agile Model for the Eco-design of Electric Vehicle Li-Ion Batteries
}

\author{
Peggy Zwolinski, Serge Tichkiewitch (1) \\ Univ. Grenoble Alpes, CNRS, G-SCOP, 38000 Grenoble, France
}

Numerous design choices need to be made at several levels when designing high-tech products: technology, processes, architecture, components, materials... and these choices need to be made in relation to the product life cycle with the corresponding experts for each stage of the life cycle. At the same time, to ensure product sustainability, a specific focus on the future potential environmental impacts is highly recommended. In this research, an agile model is proposed to help designers make decisions while monitoring environmental performance indicators of high-tech solutions. The concept of Critical Product Life Cycle Parameters had to be introduced to facilitate the eco-design of the final product. The approach is illustrated by the Electric Vehicle Li-Ion Batteries case study.

Design, Environment, Electric vehicle

\section{Introduction}

Numerous design choices need to be made at several levels when designing high-tech products: technology, processes, architecture, components, materials ... and these choices need to be made in relation to the product life cycle with the corresponding experts for each stage of the life cycle. At the same time, to ensure product sustainability, a specific focus on the future potential environmental impacts is highly recommended. Li-Ion batteries for Electric Vehicles (LIBs) are included in this category of hightech products with high environmental impacts, particularly because the Lithium battery market is growing constantly [1]. However, the literature on the environmental impact of LIBs mainly focuses on the technology used for their manufacture or on the chemistry used for recycling them [2]. But the impact distribution along the phases of the life cycle as well as the parameters that influence these impacts differ significantly depending on the cathode materials and the possible end-of-life treatments.

The challenge is therefore to highlight, throughout the design process, the key parameters on which battery designers can act in order to reduce environmental impacts and thus choose the best compromise in terms of technologies or technical solutions. In order to do this, an agile product model that incorporates an environmental view is proposed. This view, based on life-cycle analysis, highlights the critical product parameters (those that most influence environmental impacts) associated with each battery technology. These parameters are then reflected in the different experts' views (in the case of batteries: technologist, manufacturer, recycler, performance, and cost views), giving valuable information to assist the different experts in their design choices. Indeed, since a key parameter appears in an expert view, the expert knows that it can influence at least one of the main environmental impacts and is able to identify whether other actors are concerned by this parameter. Thus, the proposed representations allow for the simultaneous study of different product technologies with a high visibility of the associated impacts, which in turn enables a real consideration of the environmental dimension in design.
The first part of this paper describes how the Critical Project Life Cycle Parameters (CPLCPs) can be identified for use in an environmental view during the design process. This environmental view is presented in the second part, in relation to other experts' views. The third part illustrates the uses of the proposed agile model by designers who want to optimize a product or to make decisions considering different LIB technologies.

\section{Identification of Critical Project Life Cycle Parameters}

Life Cycle Analysis (LCA) provides environmental indicators but gives no direct clues about reducing the environmental footprint of a project [3]. It is necessary to connect the environmental indicators to the project parameters [4]. However, selecting the relevant environmental indicators and project parameters is not straightforward because of a lack of expert knowledge [5]:

- No rules are established and the know-how is not shared between practitioners;

- The selection is conducted by experts that are not always connected to the project field or who have no experience in LCA.

Our approach aims to standardize the method of connecting the environmental indicators to the project parameters based on effective and efficient decisions. This approach uses the concept of environmental hotspots to identify key parameters as powerful levers for reducing the impacts, and modifiable parameters to identify powerful levers in project decision making. The objective is to provide designers with a list of Critical Project Life Cycle Parameters (CPLCPs).

\subsection{Environmental hotspots}

The most important (or potentially highest) environmental impacts estimated by an LCA are called "environmental hotspots" [6]. They have to be identified in two dimensions: over the whole life cycle of the product and within each life cycle stage. The elements that contribute to the hotspots have to be identified to serve as levers to decrease the overall environmental load. These contributors can be identified at the Life Cycle Inventory level as: resources, waste, products, components, substances, processes ... 
If a clear contributor is identified in relation to an environmental "hotspot" or "potential hotspot" then the key parameters characterizing this contributor have to be highlighted. Therefore, the hotspot analysis in an LCA is not just about showing the relative contributions of life cycle stages or product elements to the different environmental indicators. A detailed and complex analysis must be carried out to understand the individual emissions and resource uses behind the scores and their level of influence. Three different cases have been identified:

- A hotspot identified because of a high score for a single impact, caused by a single contributor: for example, the GWP associated with a project is high and it is shown that the energy mix induces $30 \%$ of the GWP indicator when other contributors induce less than $5-10 \%$. Therefore, the energy mix is potentially a contributor and the parameter "country for the mix" is probably a key parameter for consideration in the project.

- A hotspot identified because the contributor has an effect on many different environmental impacts. In the EV LIB example, the energy mix can also be considered as a contributor for this second type of hotspot because it has a strong influence on many different indicators (ozone layer, acidification, human toxicity, and eutrophication).

- A hotspot identified because the environmental impacts are very sensitive and potentially high when a contributor is modified. In the EV LIB example, changes in the energy mix parameters (types of energies and quantities) have a strong influence on the environmental impacts.

\subsection{Project parameters}

A project parameter is an essential item of data for the understanding of a project and its life cycle characteristics during an environmental improvement process. Project parameters are related to products, processes and use behaviors [7] and could be for example the product's geometry, material, lifetime or performance, a manufacturing process, the transportation strategy or end-of-life treatment, etc. The values of these parameters are decided by the stakeholders that contribute to the project at any time, and modification of them leads to modification of the system and of the final solution.

Some of those parameters can be easily modifiable in practice, because project members directly manage them. Others cannot be modifiable or could be modifiable only after introducing new members into the project, because of the field of expertise and responsibility of those involved. Indeed, the life cycle experts (raw material providers, manufacturers, users, recyclers, etc.) have limited power of action and are only able to decide on a small number of the overall project parameters. These parameters have been called "modifiable parameters": they indicate the level of control that the project manager has over the emissions and resource utilizations. In the example of LIBs, the copper causes an environmental hotspot because it generates high environmental impacts compared to other contributors and this is true for several indicators ([8], [9], [10]). So, the energy mix to produce the copper for the anode current collector, the quantity of copper, and the amount of energy used in the copper-based component manufacture are identified as key parameters at the origin of environmental hotspots. Here, the energy mix of the specific geographical area is not modifiable by any expert involved in the project, but the quantity of copper and the quantity of energy used in the product manufacture can be optimized by the different actors in the product life cycle. Therefore, efforts to improve the environmental impacts of the project solution have to be focused on those key modifiable parameters, particularly if it is not possible to change the place of manufacture of the batteries in order to change the local energy mix.
The second important characteristic of the project parameters is their capacity to be shared. A shared parameter is a parameter that concerns different product life-cycle stakeholders. It indicates the level of dependency of the project actors on the parameter. For example, a battery recycler and a battery manufacturer are interested in the product material for different reasons: economic, legislative, performance, etc. In many cases, they have to find a compromise if the evolution of the parameter value causes positive impacts for one and negative impacts for the other.

\subsection{Critical Project Life Cycle Parameters}

Therefore, among the parameters of a project, there are key parameters (having a significant and proven influence on environmental indicators), modifiable parameters (resulting from a decision made by at least one of the project group members) and shared parameters (affecting more than one project member if their value changes).

From a practical point of view, the key and modifiable parameters, which are both at the origin of hotspots and also central to project decision-making, are called Critical Project Life Cycle Parameters (CPLCPs). Efforts to improve the environmental performance of the project have to be focused on these CPLCPs. This set is often small but is very significant because it provides the project group with the means to manage environmental impacts easily and directly.

\subsection{CPLCP identification for EV LIBS}

In order to illustrate the approach presented, two LCAs of EV LIBs were carried out. One for LFP LIBs (LiFePO4) and the other for NMC LIBs ( $\mathrm{LiNi}_{1 / 3} \mathrm{Co}_{1 / 3} \mathrm{Mn}_{1 / 3}$ ). These two LIBs differ in terms of their chemical composition and their performance but are used for the same application. Full details about LCA hypothesis and inventory can be found in [11]. The chosen FU is based on the total amount of energy stored (and released) during a typical use for EV applications (337 MWh). All the life cycle stages were considered, as recommended by [12], including a recycling process at the end of life. Environmental indicators were calculated using the CML2001 baseline method, except for the non-renewable energy indicator, which was calculated using the IMPACT 2002+ method. The hotspots and then the contributors identified are the same for both battery technologies: energy mix of electricity at the manufacturing plant, energy consumption during use phase, anode, cathode and end-of-life treatment. To establish the link between a hotspot and the corresponding CPLCPs, a closer look at the LCA results was required. Here, a recursive approach using LCA of sub-parts of the system (or its life cycle) was used to identify which project parameters contribute most to the hotspot. Some of the CPLCPs obtained were already identified in the literature: dry room energy consumption and dry room location (in relation to the energy mix of electricity at the manufacturing plant), energy density and energy efficiency of the battery (in relation to energy consumption during the use phase) and location of use (in relation to the energy mix during the use phase), recovery rate of active material and amount of active material (in relation to end-of-life treatment). Moreover, the proposed approach allows the identification of other CPLCPs: amount and recycling rate of copper (in relation to anode current collector) and amount of NMP solvent (in relation to anode and cathode materials). The results indicate that CPLCPs have different effects depending on LIB chemical composition: as presented in bold in Table 2, the amount of active material and its recovery rate influence different environmental impacts, involving different stakeholders. For example, the active material of NMC batteries is of opposing interest to the manufacturer and the recycler (because of the price of the metals it contains) whereas the active material of LFP batteries is of no interest to the recycler. 
Table 2 Contribution of identified CPLCPs for LFP and NMC Batteries

\begin{tabular}{|c|c|c|c|c|c|c|c|c|c|c|c|c|}
\hline 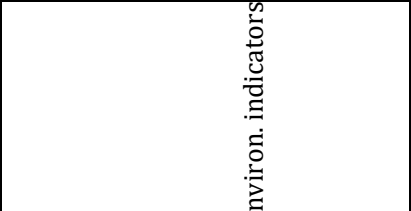 & 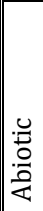 & $\begin{array}{l}\frac{0}{0} \\
.0 \\
\frac{0}{0} \\
\frac{0}{0}\end{array}$ & 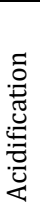 & & 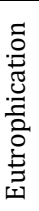 & & 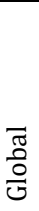 & 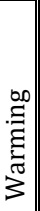 & 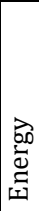 & 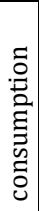 & 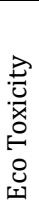 & \\
\hline $\begin{array}{l}0 \\
0 \\
0\end{array}$ & $\sum_{z}^{U}$ & 号 & $\sum_{z}^{u}$ & 足 & $\sum_{z}^{u}$ & 足 & $\sum_{z}^{U}$ & 足 & $\sum_{z}^{U}$ & 空 & $\sum_{z}^{u}$ & 空 \\
\hline Dry room energy & $\mathrm{X}$ & $\mathrm{X}$ & $\mathrm{X}$ & $\mathrm{X}$ & $\mathrm{X}$ & $\mathrm{X}$ & $\mathrm{X}$ & $\mathrm{X}$ & $\mathrm{X}$ & $\mathrm{X}$ & & $\mathrm{X}$ \\
\hline Dry room location & $\mathrm{X}$ & $\mathrm{X}$ & $\mathrm{X}$ & $\mathrm{X}$ & $\mathrm{X}$ & $\mathrm{X}$ & $\mathrm{X}$ & $\mathrm{X}$ & $\mathrm{X}$ & $\mathrm{X}$ & & $\mathrm{X}$ \\
\hline Energy density & & & & & & & & & $\mathrm{X}$ & $\mathrm{X}$ & & \\
\hline Energy efficiency & & & & & & & & & $\mathrm{X}$ & $\mathrm{X}$ & & \\
\hline Location of use & & & & & & & & & $\mathrm{X}$ & $\mathrm{X}$ & & \\
\hline Amount of copper & & & $\mathrm{X}$ & $\mathrm{X}$ & & & & & & & $\mathrm{X}$ & $\mathrm{X}$ \\
\hline Recycling rate of copper & & & $\mathrm{X}$ & $X$ & & & & & & & $\mathrm{X}$ & $\mathrm{X}$ \\
\hline Amount of NMP & & & & & $\mathrm{X}$ & $\mathrm{X}$ & & & & & & \\
\hline Amount of active material & & & $\mathbf{X}$ & & & $\mathbf{X}$ & & & & & $\mathbf{X}$ & \\
\hline Recovery rate of active material & & & $\mathbf{X}$ & $\mathbf{X}$ & $\mathbf{X}$ & & & & & & $\mathbf{X}$ & $\mathbf{X}$ \\
\hline
\end{tabular}

\section{The environmental view in the agile model}

Figure 1 gives a partial representation of the agile product model for LFP LIBs. It partly shows the different views taken into account in the project, with the product characteristics attached to each expert: a technological view, with the constituent components of the product and their characteristics (mass, material, etc.); a manufacturing view that describes manufacturing processes and their characteristics (capacity, energy consumption, etc.); a recycling view that also describes the processes at this stage of the life cycle (materials to be recovered, recyclability rate, recycling processes); an economic view that presents the costs and revenues associated with the life cycle stages of the product (e.g. manufacturing costs, energy costs, material prices or potential recycling revenues); a performance view that indicates the service specificities delivered by the product (lifetime, power, aesthetics, etc.) and the environmental view that shows the impact levels and key parameters associated with these impacts. In general, these five views are sufficient to describe high-tech products, but other views can be included to further model a particular product. The objective here is to incorporate the environmental knowledge obtained through the LCA into the various views so it can be taken into consideration during the design process [13].

The environmental view shows the selected impact categories and the level of impact measured in the corresponding unit, as well as the key parameters - those that contribute to more than $10 \%$ of the total impact.

Figure 1. The agile model for LFP LIBs (partial views and links)

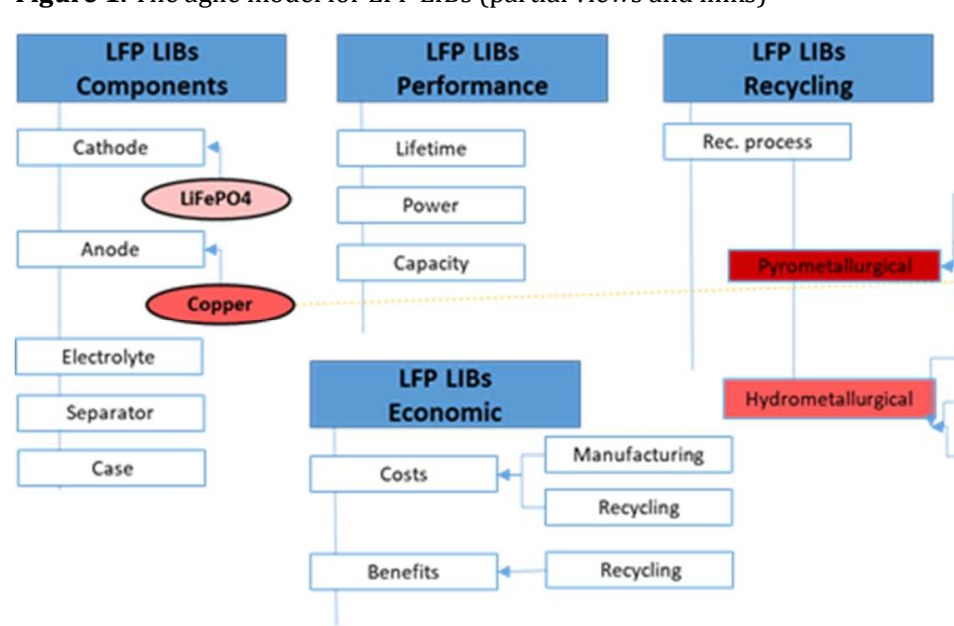

Figure 2 The environmental view for the LFP and NCM LIBs technologies

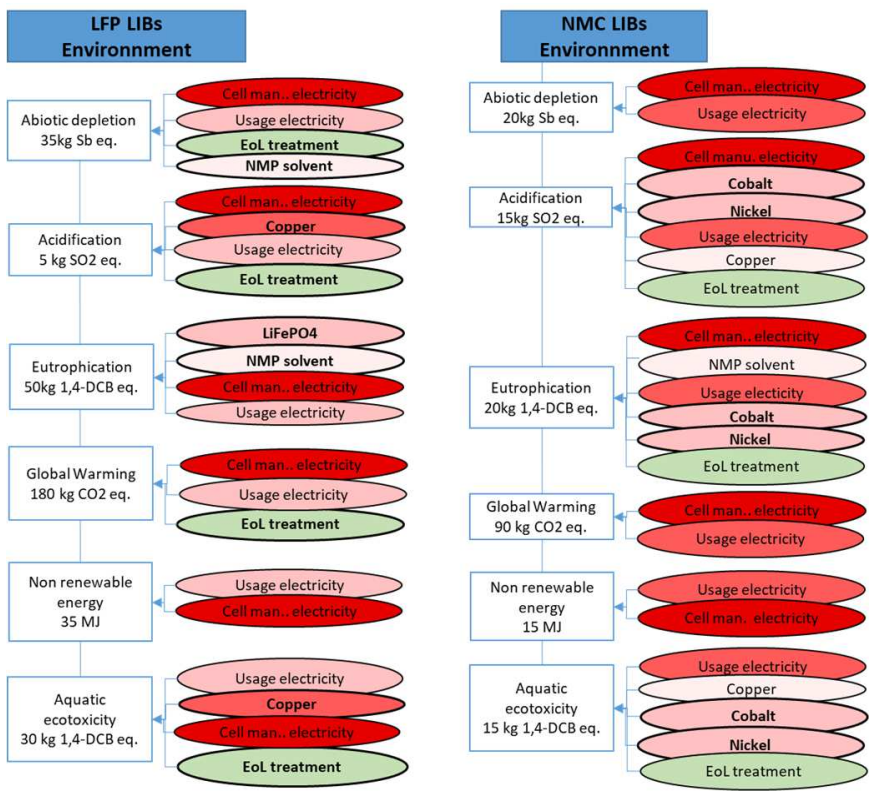

These key parameters associated with each impact are classified from most influential to least. For example, regarding the abiotic depletion (ADP) of the NMC LIB, only two parameters were identified: energy consumption for manufacturing and use. A color scale is defined and one color is assigned to each key parameter according to its frequency and degree of involvement in the different impacts (red for positive impacts and green for negative). A shade of red is thus attributed to each key parameter, the darkest color being attributed to the key parameter generating the most impacts (figure 1 and 2). These colors are also applied to the parameters in the different experts' views and links can be plotted between them to identify shared parameters and consequently all the actors that will be concerned by the evolution of any given parameter. Uncolored parameters are non-key parameters. In addition, parameters that can be modified by the design team are displayed in bold (cf. end-of-life treatment or materials). One can see here, that the environmental impacts for LFP LIBs are higher in all impact categories, except for the eutrophication. To decrease those impacts, designers could modify the solvent or the LiFePO4 (bold) but because those components are in light red, this will probably not decrease a lot the global impact of the product. In order to decrease the global impact of the two LIBs, designers should discuss with manufacturers to decrease or transform the electricity used for the battery manufacturing.

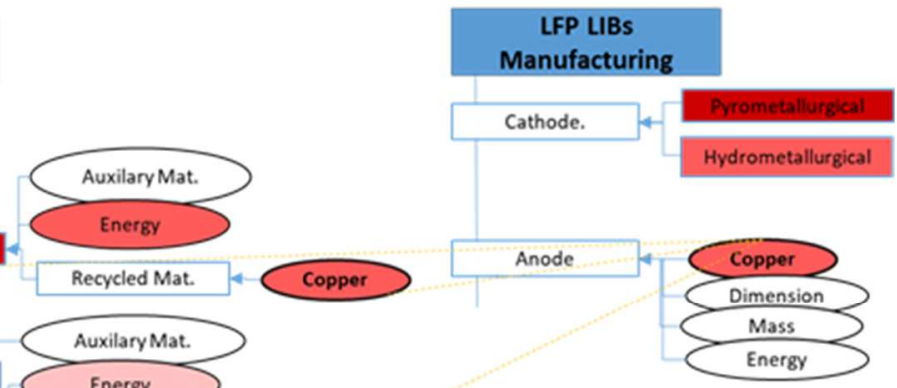


To reduce the overall impact, the designer must observe the color code of the key parameters and work as a priority on those in dark red (which contribute significantly to the overall impact). To reduce the impact on a specific indicator, the designer must ignore the color code and observe the classification of the key parameters associated with the indicator. In both cases, the designer is able to monitor the evolution of quantitative impacts through each impact level.

\section{Case study: Eco-design of Electric Vehicle Li-Ion Batteries}

When using this agile model in a case study, the designers' objective was to optimize a battery from an environmental point of view while maximizing its recyclability. Based on the impacts and parameters identified in the LFP LIB agile model, new options and modifiable parameters were proposed: the LFP manufacturing process ("hydrothermal" replaced with a "solid-state" process), the manufacturing solvent (NMP solvent replaced with an aqueous solvent), the manufacturing location (France instead of China), the type of end-of-life process (pyrometallurgical or hydrometallurgical) and the copper recovery rate (85\% or $95 \%)$. However, despite the different configurations tested, it was not possible to reach an acceptable compromise in terms of economic and environmental costs. The LFP LIB technology shows a very significant end-of-life constraint: the recycling process is barely cost-effective and the recycling rates imposed by the standard are not verified. Therefore, very few levers exist to improve the overall impact.

The designers decided to switch to another battery technology: NMC batteries. The environmental view for NMC batteries (Figure 2) shows that new key parameters have emerged and others have changed color, because they influence certain impacts to a greater or lesser degree. For example, "NMP solvent" and "hydrometallurgical end-of-life treatment" are no longer key parameters for the Abiotic Resource Depletion indicator (ADP), and end-of-life treatment benefits certain indicators. In fact, the NCM cathode active material appears as a key parameter because it is involved in several indicators, due to the presence of heavy metals such as cobalt and nickel, which are high-impact materials.

The benefits of the agile model can be clearly seen, because the modifiable key parameters of the NCM LIB technology are very different from those identified with the previous LFP LIB technology. For example, the manufacturing process is no longer a modifiable parameter, while the end-of-life treatment is modifiable. Parameter comparison does not make sense here, since the parameter configurations between Li-ion technologies are not the same: they disappear or are no longer influential. Nickel is an example of a parameter that disappears when the technology changes from NCM to LFP. With the agile model, the designers were able to fully appreciate the impact of the change in the Li-ion technology parameter. They found that many design parameters were indirectly changed or "disappeared" from the model.

Finally, the NCM LIB technology guarantees the profitability of recycling and compliance with standards. Moreover, despite more powerful materials, this technology is generally less impactful than the first. The environmental impact of heavy metals has been compensated by other actions on other modifiable key parameters.

\section{Conclusion}

To decrease the environmental impacts of the use of LIBs for Electric Vehicles, it is necessary to identify the Critical Levers that could be influenced by the project members. The use of the agile model integrating those CPLCPs, in this particular example of Liion batteries, shows the importance of highlighting key and modifiable parameters. These are of particular interest to the designers, since they know that their modifications will have a direct effect on environmental impacts. In fact:

- Simple analysis of the environmental impact contributors is not sufficient to highlight the most relevant levers to help make decisions within the project. In our LIB case study, this level of analysis could lead to the same conclusion concerning the two different batteries. However, it has been shown that clear differences exist between the two solutions considered.

- Hotspots and/or contributors and/or key parameters could be hidden because of a partial analysis of the results (too focused on one life cycle stage) or because no sensitive analysis is performed. In the case of LIBs, the percentage of copper recovered could have been hidden if the sensitive analysis of the contributors had not been conducted.

- Key parameters could also be neglected, either because they are distributed across several indicators, or they are only identified in one solution or they influence different impacts. This is the case for the active material in LIBs that generates hotspots in different impact categories.

This approach provides the team of experts with a list of CPLCPs that are the main levers for acting on environmental impacts. These CPLCPs are represented in an agile model to better integrate them during the design process and to address the right expert(s) to ask for modifications. Once implemented in a software, this approach will provide designers with a powerful tool to control design parameters in relation to environmental impacts. While adapting the modeling in the different views, it will help decision making for every lifecycle stakeholder, including the end-users with the performance view. Consequently, an integrated ecodesign approach can be developed, involving all the necessary project stakeholders, who will have clear levers to influence the environmental issues.

\section{References}

[1] 2012 Minerals Yearbook, Lithium, U.S. GEOLOGICAL SURVEY, 13p 2013

[2] Dunn J.B., Gaines L., Barnes M., Sullivan J., Wang M.Q., Material and energy flows in the materials production, assembly and end-of-life stages of the automotive lithium-ion battery life cycle, Argonne National Lab., 2012

[3] Bonou A., Olsen S.I., Hauschild M.Z., Introducing life cycle thinking in product development - A case from Siemens Wind Power, CIRP Annals, 64/1, pp 45-48, 2015

[4] Pegoretti dos Santos T., Mathieux F., Evrard D., Brissaud D., de França Arruda J.R., Use of recycled natural fibres in industrial products: A comparative LCA case study on acoustic components in the Brazilian automotive sector, Resources, Conservation \& Recycling, 84, pp 1-14, 2014

[5] Bey N., Hauschild M.Z., McAloone T.C., Drivers and barriers for implementation of environmental strategies in manufacturing companies, CIRP Annals, 62/1, pp 43-48, 2013

[6] Hauschild M.Z., Jeswiet J., Alting L., Design for environment - Do we get the focus right?, CIRP Annals, 53/1, pp 1-4, 2004

[7] Cor E., Domingo L., Brissaud D., Zwolinski P., A protocol to perform usage oriented ecodesign, CIRP Annals, 63/1, pp 169-172, 2014

[8] Dunn J.B., Gaines L., Sullivan J., Wang M.Q., Impact of recycling on cradle-to-gate energy consumption and greenhouse gas emissions of automotive lithium-ion batteries., Environ. Sci. Technol., 46, 22, pp 12704-12710, 2012

[9] Notter D.A., Gauch M., Widmer R., Wäger P., Stamp A, Zah R., Althaus H.-J., Contribution of Li-ion batteries to the environmental impact of electric vehicles, Environ. Sci. Technol., 44, 17, pp 6550-6556, 2010

[10] Ellingsen L.A.-W., Majeau-Bettez G., Singh B., Srivastava A.K., Valøen L.O., Strømman A.H., Life Cycle Assessment of a Lithium-Ion Battery Vehicle Pack, J. Ind. Ecol., vol. 18, pp. 113-124, 2014

[11] Lorente D.B., Proposition d'un modèle produit agile pour l'écoconception: application aux batteries Li-ion, PhD thesis, Univ. Grenoble Alpes, 2016, http://www.theses.fr/s95817

[12] Lorente D.B., Mandil, G., Svecova, L., Thivel P-X., Zwolinski P., Life Cycle and Sustainability. In Lithium Process Chemistry - Resources, Extraction, Batteries, and Recycling, edited by A. Chagnes and J. Swiatowska, pp. 269-288, 2015

[13] Dufrene M., Zwolinski P., Brissaud D., An engineering platform to support a practical integrated eco-design methodology, CIRP Annals, 62/1, pp 131-134, 2013 